\title{
A Multi-step Heart Rate Prediction Method based on Physical Activity using Adams-Bashforth Technique
}

\author{
Feng Xiao, Ming Yuchi, Ming-yue Ding, Jun Jo and Jong-Hwan Kim
}

\begin{abstract}
Physical activity (PA) is commonly recognized to directly influence changes in heart rate (HR). HR prediction based on PA can be a useful tool in medical research and monitoring in a clinical setting. In our previous works, predictors with high accuracy were designed. However, the HR could only be predicted in single time steps. In this study, a multi-step HR prediction method is proposed. Firstly, the HR prediction problem was converted into an Initial-Value Problem for Ordinary Differential Equation (IVPODE). Then the Adams-Bashforth method was used to implement multi-step prediction. Only HR at the initial time step and PA signals are needed. Experiments were conducted based on the real-life signals from a healthy male. Predicted HR can approximately trace the actual HR in a long time step. The results show the potential of this proposed method.
\end{abstract}

\section{INTRODUCTION AND MOTIVATION}

Heart rate is one of important physiological parameters and can be easily obtained. It is commonly used in medical/exercise research and clinical monitoring. It has been found that many factors affect changes in HR, such as: physical activity (PA), mental activities and the individual's surroundings. Among these factors, it is commonly recognized that PA affects the changes in HR directly. However, only a few works have described how PA influence HR: Pawar et al. [1] presented a body movement activity detection system which was based on an ECG signal, but without HR. Meijer et al. [2] built a linear relationship between HR and body movements, but their experiments were implemented under specific conditions and the body movement was recorded as the counted number of activities, which did not appropriately reflect the actual PA. Bezerianos et al. [3] introduced RBF network based methods for the assessment of the dynamics of the heart rate variability signal, but the HR prediction was based on pharmacological effects.

In our previous works [4], [5], HR predictors based on PA were designed to discover how PA affects HR. Feedforward Neural Networks (FFNN) were adopted to perform the prediction. In the experiments, the subject was equipped with a portable HR and PA monitor and performed normal daily activities, without any special routine or restriction. $\mathrm{HR}$ and PA signals were recorded and preprocessed to be

This research was sponsored by the Queensland International Fellowships Program, Australia.

Corresponding Author: Ming Yuchi (Email: m.yuchi@gmail.com)

F. Xiao, M. Yuchi and M. Ding are with Faculty of Life Science and Technology, Huazhong University of Science and Technology, Wuhan 430074, China

J. Jo is with the School of Information and Communication Technology, Griffith University, Queensland, Australia

J-H. Kim is with the Department of Electrical Engineering, Korea Advanced Institute of Science and technology, Daejeon, Korea

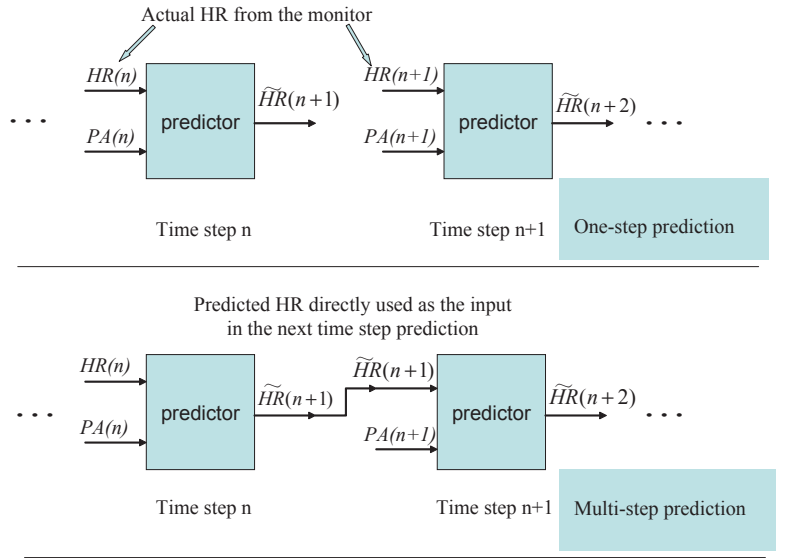

Fig. 1. A comparison between one-step and multi-step prediction methods: $\mathrm{HR}$ with a wave line above is the predicted HR while actual HR does not have a wave line

synchronous. The synchronous HR and PA signals at time step $n$ were set as the inputs for the FFNN predictor, and the output of the predictor was HR at the next time step, $n+1$. Experimental results showed that the mean absolute error of the predicted heart rate could be restricted to a small range. However, these works used the one-step prediction strategy (Fig. 1) which meant that HR could be predicted in only one time step (from $n$ to $n+1$ ).

The main motivation of this work is to provide a novel method that can predict HR over a long time period at a certain precise level. Predicted HR at time step $n$, instead of the actual HR at time step $n$ in the previous works, is used to predict HR at the next time step, $n+1$ (multi-step prediction). Fig. 1 shows the difference between one-step and multi-step prediction. The proposed multi-step prediction is as follows: firstly, the multi-step prediction problem is converted into an Initial-Value Problem for Ordinary Differential Equations (IVPODE). FFNN [6], [7] was chosen as the function approximator of the Ordinary Differential Equations (ODE) in the IVPODE. The Adams-Bashforth technique [8], [9] is used to perform the prediction by solving the ODE.

\section{RESEARCH METHOD}

\section{A. Research Model}

To investigate the relationship between the HR and PA, all the signals had to be sampled simultaneously. A portable HR and PA monitor from Alive Technologies was used. The monitor measured and recorded the subject's ECG and PA (3-D acceleration) signals and determined the HR from the 


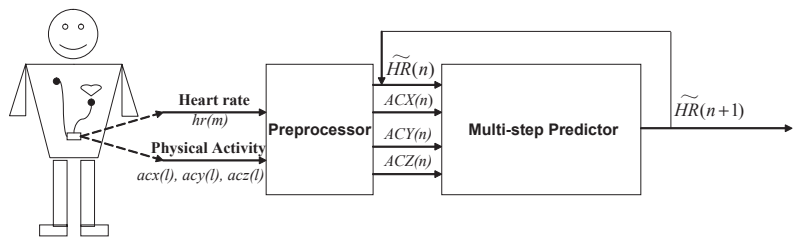

Fig. 2. The Block diagram of the whole system in this work: HR with a wave line above is the predicted HR while actual HR does not have a wave line

TABLE I

Data Specifications of Alive Heart Monitor

\begin{tabular}{|c|c|c|}
\hline Signal & $E C G$ & Accelerometer \\
\hline Channels/Axis & Single Channel & 3 Axes \\
\hline Resolution & 8 bits & 8 bits \\
\hline Sampling Rate & $1 \mathrm{samples} / \mathrm{sec}$ & $75 \mathrm{samples} / \mathrm{sec}$ \\
\hline Dynamic Range & $-2.66 \mathrm{mV}-2.66 \mathrm{mV}$ & $-2.7 \mathrm{~g}-2.7 \mathrm{~g}$ \\
\hline Bandwidth & $0.5 \mathrm{~Hz}-90 \mathrm{~Hz}$ & $0 \mathrm{~Hz}-20 \mathrm{~Hz}$ \\
\hline
\end{tabular}

ECG in real-time. The left section of Fig. 2 shows the subject (user) wearing the monitor.

The middle section of Fig. 2 is the preprocessor, it converted the recorded signals into four synchronous signals for the coming prediction. The details of the preprocessor will be described in Section II-B.

The right section of Fig. 2 is the multi-step predictor. The multi-step prediction problem was converted into an IVPODE. The details of the predictor's mathematic model (IVPODE) will be presented in Section II-C.

\section{B. Signal Sampling and Preprocess}

Most studies of HR are based on the experimental data collected under specific conditions and/or environments, whereas this research was conducted with the data collected from normal daily activities, without any pre-planned routine. Consequently, a portable device was needed, which can monitor and record the HR and PA signals simultaneously with relatively high accuracy. A current commercial product, the Alive Heart Monitor (AHM), was chosen for our experiments. The collected data can be saved to an internal SD memory card or transmitted to PC, smart phone or PDA using Bluetooth in real time. The data specification of the AHM is shown in Table I.

The sampling rates of HR $(h r(m))$ and acceleration $(a c x(l), a c y(l), a c z(l))$ are set differently in the AHM, 1 sample/sec and 75 samples/sec, respectively. However, the inputs of the predictor are normally required to be sequences with the same sampling rate. Here, $h r(m)$ and $a c x(l)$, $a c y(l), a c z(l)$ are converted into four synchronized sequences $H R(n)$ and $A c x(n), A c y(n), A c z(n)$ through a processing period $\tau$.

Assume the whole recording period is $T$, the recorded data on each signal channel are evenly divided into $\mathrm{N}$ segments, where each segment has the length of $\tau$ :

$$
N=\operatorname{floor}(T / \boldsymbol{\tau})
$$

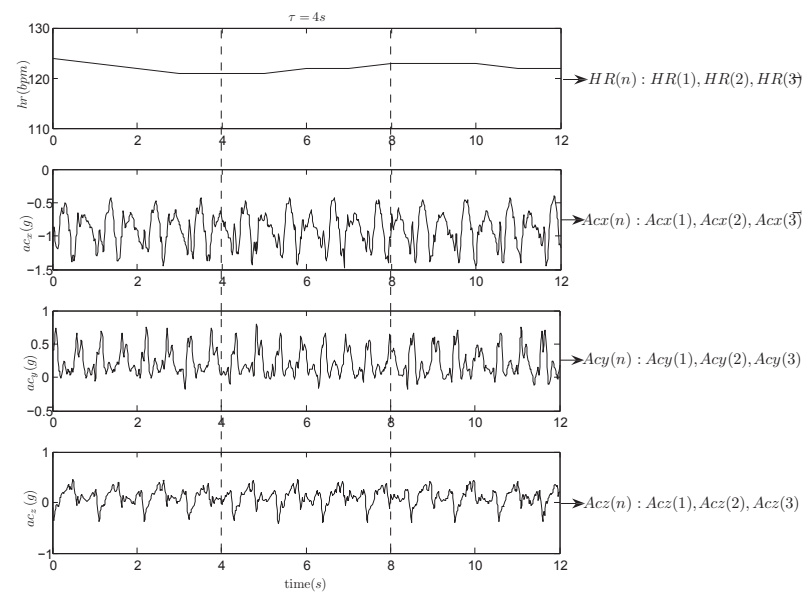

Fig. 3. An example of the signal preprocess

and floor $(x)$ rounds $x$ to the nearest integer towards minus infinity. The left part of Fig. 3 shows an example with $\mathrm{T}=12 \mathrm{~s}$ and $\tau=4 \mathrm{~s}$. The recorded data are divided into $\mathrm{N}=12 / 4=3$ segments on each channel.

Each HR segment has $N_{h r}$ samples:

$$
N_{h r}=\text { SamplingRate }_{h r} \times \tau,
$$

and each acceleration segment has $N_{a c}$ samples:

$$
N_{a c}=\text { SamplingRate }_{a c} \times \tau .
$$

When $\tau=4 \mathrm{~s}$, HR segment has 1 sample/s $\times 4 \mathrm{~s}=4$ samples $\left(N_{h r}\right)$, and each acceleration segment has 75 samples $/ \mathrm{s} \times 4 \mathrm{~s}$ $=300$ samples $\left(N_{a c}\right)$. Then, the $n t h(n=1, \ldots, \mathrm{N}) h r$ segment is converted into $\operatorname{HR}(\mathrm{n})$ with the equation 4 , and the nth acx, acy, acz segments are converted into $A c x(n)$, $\operatorname{Acy}(n), \operatorname{Acz}(n)$ with the equation 5.

$$
\begin{aligned}
& H R(n)=\frac{\sum_{m=(n-1) \times N_{h r}+1}^{n \times N_{h r}} h r(m)}{N_{h r}} ; \\
& \operatorname{Acx}(n)=\frac{\sum_{l=(n-1) \times N_{a c}+1}^{n \times N_{a c}} a c x(l)}{N_{a c}}, \\
& \operatorname{Acy}(n)=\frac{\sum_{l=(n-1) \times N_{a c}+1}^{n \times N_{a c}} a c y(l)}{N_{a c}}, \\
& A c z(n)=\frac{\sum_{l=(n-1) \times N_{a c}+1}^{n \times N_{a c}} a c z(l)}{N_{a c}} .
\end{aligned}
$$

It should be noted that the function of $\tau$ is not only to synchronize the inputs to neural network, but also to help stabilize the prediction accuracy through averaging the noises. This works well, especially when some signals have random noises. 


\section{Mathematic Basis and Structure of Predictor}

In this paper, the Adams-Bashforth technique is used for prediction. To use this technique, the prediction problem first needed to be converted into an IVPODE. The classical IVPODE is in the form:

$$
\frac{d w}{d t}=f(t, w(t)) \quad \text { for } a \leqslant t \leqslant b,
$$

subject to an initial condition:

$$
w(a)=\alpha .
$$

Formula 6 can be approximated as:

$$
\begin{aligned}
\Delta w(i)=\Delta t \cdot f(t(i), w(i)) & \text { for } N_{a} \leqslant i \leqslant N_{b}, \\
& \text { or } \quad \text { for } N_{a} \leqslant i \leqslant N_{b},
\end{aligned}
$$

subject to an initial condition:

$$
w\left(N_{a}\right)=\alpha
$$

where $\triangle w$ is the approximate value of $d w, h$ (or $\triangle t$ ) is the approximate value of $d t$, and $N_{a}, i, N_{b}$ are the corresponding discrete values of $a, t, b$ in formula 6 .

If the specific expression of the function $f(\cdot)$ is known, the values of $w$ between $N_{a}+1$ and $N_{b}$ can be calculated with the equation 8 below, which is called Euler's Method:

$$
w(i+1)=w(i)+\triangle w(i) .
$$

Formula 8 can be adopted for multi-step prediction using the initial value $w\left(N_{a}\right)$ to predict the value of $w$ from $N_{a}+1$ to $N_{b}$. However, the precise level of Euler's Method is not high enough especially when the system is nonlinear.

One possible way to increase the prediction precision is to use more previous states, like $w(i-1), w(i-2)$, to determine the approximation value of $w(i+1)$. One of the popular methods is the Adams-Bashforth method[8], [9]. The method is shown below:

(1) Adams-Bashforth Two-step Explicit Method:

$$
\begin{aligned}
w(0) & =\alpha(0), w(1)=\alpha(1), \\
w(i+1) & =w(i)+\frac{h}{2}[3 \cdot f(t(i), w(i)) \\
& -f(t(i-1), w(i-1))] .
\end{aligned}
$$

(2) Adams-Bashforth Three-step Explicit Method:

$$
\begin{aligned}
w(0) & =\alpha(0), w(1)=\alpha(1), w(2)=\alpha(2), \\
w(i+1) & =w(i)+\frac{h}{12}[23 \cdot f(t(i), w(i)) \\
& -16 \cdot f(t(i-1), w(i-1)) \\
& +5 \cdot f(t(i-2), w(i-2))] .
\end{aligned}
$$

(3) Adams-Bashforth Fourth-step Explicit Method:

$$
\begin{aligned}
w(0) & =\alpha(0), w(1)=\alpha(1) \\
w(2) & =\alpha(2), w(3)=\alpha(3), \\
w(i+1) & =w(i)+\frac{h}{24}[55 \cdot f(t(i), w(i)) \\
& -59 \cdot f(t(i-1), w(i-1)) \\
& +37 \cdot f(t(i-2), w(i-2)) \\
& -9 \cdot f(t(i-3), w(i-3))] .
\end{aligned}
$$

To use the Adams-Bashforth method for HR prediction, the HR prediction problem is first converted into the following form

$$
\triangle H R(n+1)=h \cdot f(H R(n), A c x(n), A c y(n), A c z(n)),
$$

subject to an initial condition:

$$
H R(\alpha)=h r
$$

where $h$ is the step size of the prediction.

To deal with this IVPODE (12), the specific expression of the function $f(\cdot)$ should first be known. Here, FFNN is used as the approximator of $h \cdot f(\cdot)$. Fig. 4 shows the structure of the FFNN. There are four inputs to the FFNN: predicted $H R(n)$ and $A c x(n), A c y(n), A c z(n)$ at the time step n. The output of FFNN is $\triangle H R(n)$, then the predicted $H R(n+1)$ at next time step $n+1$ can be calculated using the AdamsBashforth method. From formulas (9), (10), (11), formula (13) can be deduced:

$$
\begin{aligned}
H R(0) & =h r \\
H R(1) & =H R(0)+\triangle H R(0), \\
H R(2) & =H R(1)+\frac{1}{2}[3 \triangle H R(1)-\triangle H R(0)], \\
H R(3) & =H R(2)+\frac{1}{12}[23 \triangle H R(2)-16 \triangle H R(1) \\
& +5 \triangle H R(0)], \\
H R(n+1) & =H R(n)+\frac{1}{24}[55 \triangle H R(n) \\
& -59 \triangle H R(n-1)+16 \triangle H R(n-2) \\
& -9 \triangle H R(n-3)] .
\end{aligned}
$$

where $\triangle H R(n)$ was calculated by the trained FFNN function approximator.

Fig. 5 shows the structure of the multi-step predictor using formula (13). It is evident that only predicted HR and the PA signals are used in the predictor.

\section{EXPERIMENT}

\section{A. Experiment dataset}

In this paper, the subject was a 33 year old male with no history of heart disease. The recording time was 12 minutes $(\tau / h=4 \mathrm{~s})$. During this period, the subject wore an AHM and performed his normal daily activities. The signals during the 12 minutes were evenly separated into two parts. The first 


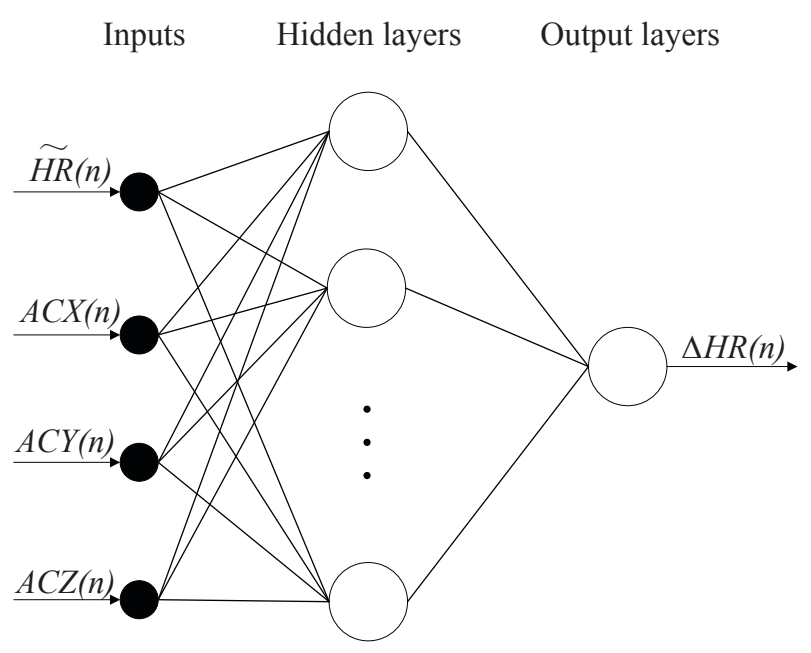

Fig. 4. Two-layer FFNN structure

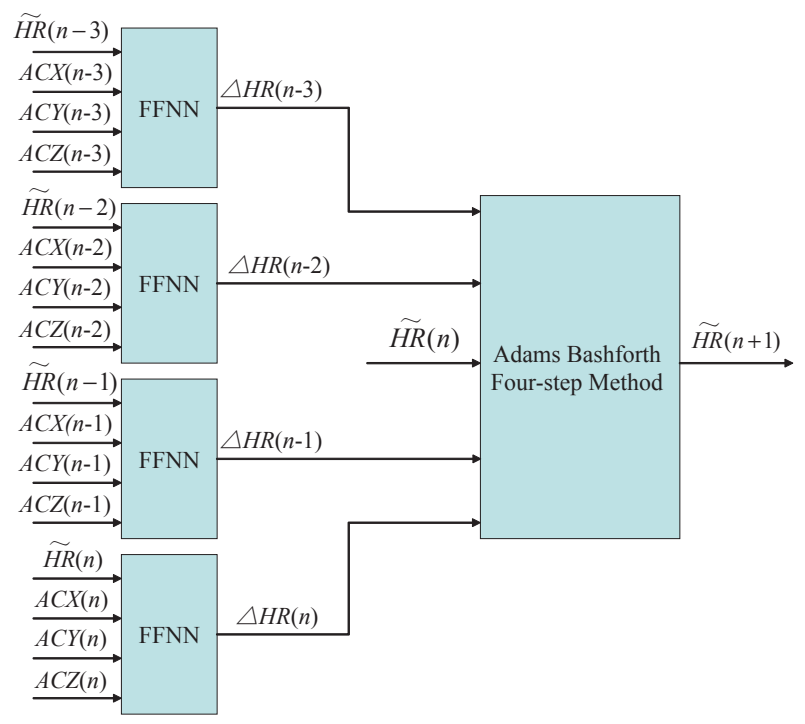

Fig. 5. The structure of our multi-step predictor: HR with a wave line above is the predicted HR while actual HR does not have a wave line.

signals $(6 \mathrm{~min})$ were adopted as the training set, which was used to train the FFNN; the remaining part of the signals (6min) were the test set, which was used to validate the trained neural network. For both the training and test sets, the total number of steps $\mathrm{N}=90$. The corresponding $H R(n)$ and $A c x(n), A c y(n), A c z(n)$ of the training set and test set were preprocessed using fomulas (4) and (5).

$\mathrm{C} / \mathrm{C}++$ was chosen as the programming language. Twolayer FFNN was selected as the ODE approximator for this experiment. The four inputs of the FFNN were $H R(n)$ and $A c x(n), A c y(n), A c z(n)$. The output layer (the last layer) had one neuron, $\triangle H R(n)$, the variance of $\mathrm{HR}$ at the time step $\mathrm{n}$. The FFNN predictor was trained using the Levenberg-Marquardt algorithm [10], [11]. According to the Kolmogorov theorem [12], the number of neurons in the hidden layer (first layer) was set to be 9 .

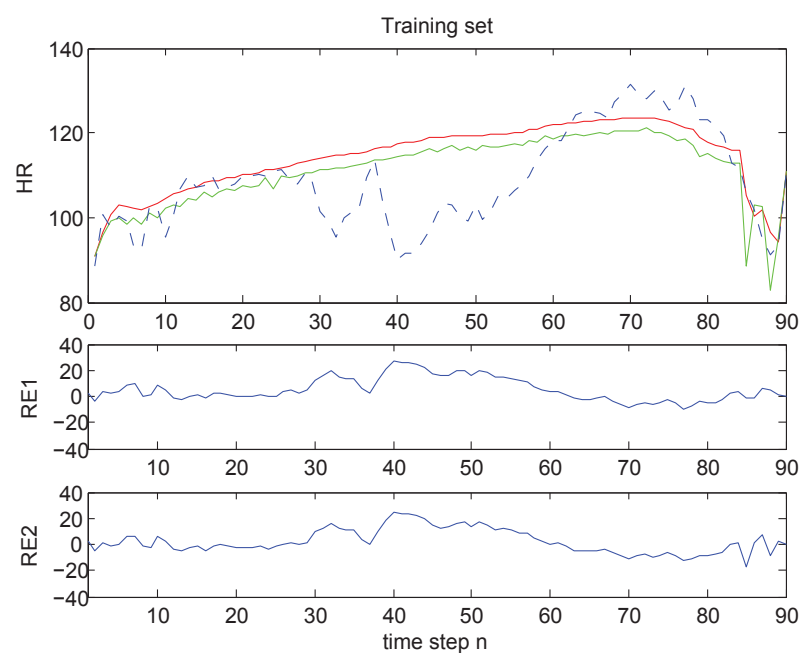

(a)

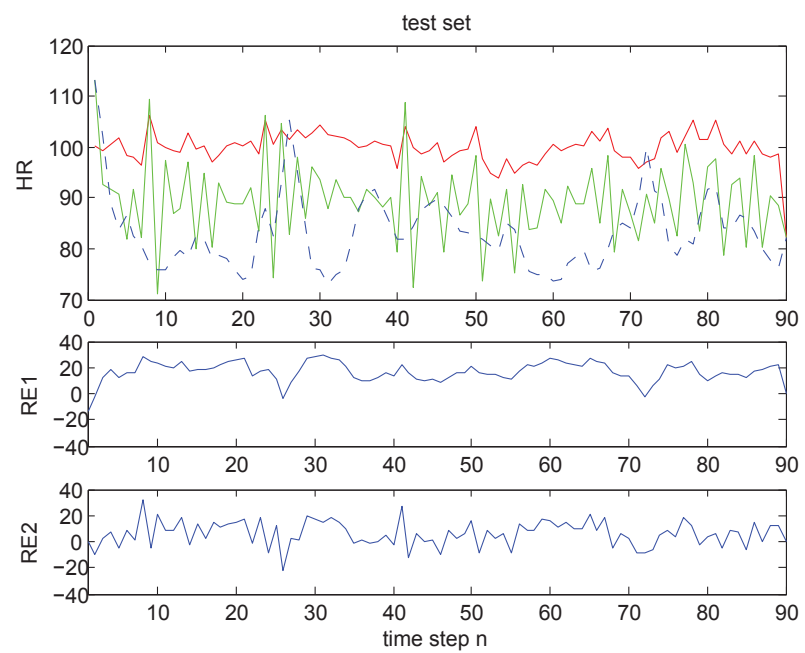

(b)

Fig. 6. Performance of the predictor. predicted $H R(n+1)$ red:Euler's Method; green:using Adams-Bashforth method; blue: actual HR(n + 1). The corresponding Residual Error(RE), RE1: the residual error between the red and the blue curve; RE2: the residual error between the green and the blue curve. (a) Training set; (b) Test set.

\section{B. Experiment Result}

The performance of the neural network predictor on the training set and test set is shown in Fig. 6. To make them easy to distinguish, the actual $H R(n+1)$ is represented by a blue dashed line, while $H R(n+1)$ predicted using Euler's Method and the Adams-Bashforth method are denoted by red and green real lines, respectively. The figures indicate that the $H R(n+1)$ can approximately trace the variance of $H R(n+1)$ on both the training set and test set after training.

The residual errors (REs) between the actual $H R(n+1)$ and the predicted $H R(n+1)$ are also shown in Fig.4. Although some REs are as large as 35 (Fig. 6(b), RE2), most of the REs are restricted to a range of about 20. The corresponding mean absolute errors (MAEs) of the training set and test set are shown in Table II. In this table, the MAEs at predicted time steps $N=10, N=20, N=40$ and 
TABLE II

The Mean Absolute Error between the actual and the PREDICTED DATA (MULTI-STEP PREDICTION)

\begin{tabular}{|c||c|c|c|c|}
\hline MAEs(training/test) & $N=10$ & $N=20$ & $N=40$ & $N=90$ \\
\hline Euler's Method & $3.048 / 16.657$ & $4.433 / 17.200$ & $5.973 / 18.152$ & $7.572 / 18.944$ \\
\hline Adams-Bashforth method & $3.347 / 9.321$ & $2.977 / 9.982$ & $5.096 / 9.721$ & $7.389 / 9.284$ \\
\hline
\end{tabular}

$N=90$ are given for comparison. It can be seen that the MAEs increased as the predicted time step rose, both on the training and test sets. It is also obvious that the performance of the predictor using the Adams-Bashforth method was better than Euler's Method. It should be noted that the results of the Adams-Bashforth method was significantly better in situations where the actual HR rose or declined sharply.

\section{Future WORK AND Discussion}

Considering that HR prediction was implemented based on the previous predicted HR and the experiments were performed on real-life data, both the REs and the MAEs of the predictor are acceptable.

To validate the feasibility of the proposed method and improve the neural network performance, further and deeper investigations should be conducted. Firstly, varied data from more subjects of varying age, gender and health level should be tested. Secondly, more tests on different system parameters, including prediction interval, total time length and sampling rate of the hardware should be implemented. Thirdly, more Neural Network structures and types are needed. The RBF is another type of Neural Network which can be used as a predictor.

The most important factors to improve this system may be the PA preprocess and the ODE multi-step solution method. More useful signals preprocessed from PA signals could also be added into the predictor as inputs. For example, the standard deviation and the gradient of PA signals. Besides the Adams-Bashforth method, multi-step methods can be considered in the future. A nonlinear multi-step method may also be a good candidate.

\section{REFERENCES}

[1] T. Pawar, S. Chaudhuri, and S. Duttagupta, "Body movement activity recognition for ambulatory cardiac monitoring," Biomedical Engineering, IEEE Transactions on, vol. 54, no. 5, pp. 874-882, May 2007.

[2] G. Meijer, K. Websterp, and H. Koper, "Assessment of energy expenditure by recording heart rate and body acceleration," Medicine and Science in Sports and Exercise, vol. 21, pp. 343-347, 1989.

[3] A. Bezerianos, S. Papadimitriou, and D. Alexopoulos, "Radial basis function neural networks for the characterization of heart rate variability dynamics," Artificial intelligence in medicine, vol. 15, no. 3, pp. 251-234, March 1999.

[4] M. Yuchi and J. Jo, "Heart rate prediction based on physical activity using feedforwad neural network," Hybrid Information Technology, International Conference on, vol. 0, 2008.

[5] F. Xiao, M. Yuchi, M. Ding, J. Jo, and W. Hou, "A research of physical activitys influence on heart rate using feedforward neural network," Neural Network, International Symposium on, vol. 0, 2009.

[6] M. Hagan, H. Demuth, and M. Beale, Neural network design. PWS Publishing Co. Boston, MA, 1997.

[7] K. Hornik, M. Stinchcombe, and H. White, "Multilayer feedforward networks are universal approximators," Neural Netw., vol. 2, no. 5, pp. 359-366, 1989.
[8] R. Adams, Sobolev spaces. Academic Press, New York, 1975.

[9] R. Burden and J. Faires, Numerical Analysis. Academic Press, New York, 1975.

[10] K. Levenberg, "A method for the solution of certain non-linear problems in least squares," Quart. Appl. Math., vol. 2, pp. 164-168, 1944.

[11] D. Marquardt, "An algorithm for least-squares estimation of nonlinear parameters," SIAM J. Appl. Math., vol. 11, pp. 431-441, 1963.

[12] S. Haykin, Neural Networks, A Comprehensive Foundation. Macmillan College Publication. NewYork, US, 1994. 